\title{
Prognosis of Diabetes using Neural Network, Fuzzy Logic, Gaussian Kernel Method
}

\author{
Tejashri N. Giri \\ Department of computer Engineering \\ Imperial College of Engineering and Research, \\ Pune India
}

\author{
Satish R. Todmal \\ Department of computer Engineering \\ Imperial College of Engineering and Research, \\ Pune India
}

\begin{abstract}
In Today's world there is an increase in the prevalence of diabetes mellitus and therefore the disease is recognising as a major global public health problem.medical data mining extracts hidden patterns from medical data.

This is to design system for diabetes prediction.. The soft computing technique is most useful and powerful technique used for diagnosis purpose. The proposed system a novel approach for diagnosis of diabetes which has two stages to predict the diabetes status. Initial Stage we are using Gaussian kernel function which help to distribution of data and second stage adopt two computational intelligence and knowledge engineering technique such as fuzzy logic and neural network. The benefit applying these is that accuracy of prediction rate will be higher than most of the suggested system for predicting the occurrence of diabetes mellitus. The dataset used for the Experimental is based on Pima Indian Dataset from University of California.
\end{abstract}

\section{Keywords}

Diabetes; Fuzzylogic; Neural network; Gaussian kernel function.

\section{INTRODUCTION}

Now day's computer technology is used in medical field to diagnose the disease. There is an increases prevavalances of diabetes mellitus is major global health problem. There are basically three main types of diabetes in type 1diabetes the insulin is failed to produce in body, Type 2 diabetes cells in human body failed to use insulin properly and gestational that is presence of high blood sugar level during pregnancy data mining technique is used for testing the accuracy of predicting diabetes status in this paper we use artificial neural network use for classification and clustering for improve the accuracy of classification gaussion kernel function applied on dataset which help to improve classification. Artificial Neural network used for pattern recognition. Fuzzy logic is suitable tool when problem has dynamic behaviour which helps to improve the prediction of system.

\section{Limitation of current System}

- The existing system had number of issues unaddressed and also prediction rate was not very appreciable.

- $\quad$ Needed database good quality

- $\quad$ Some needed Standardize ontology

- Some system fails to show the performance

- The new system is proposed that improve prediction rate.
Proposed framework consists of neural network and fuzzy logic technique. Gaussian kernel function used to improve the accuracy of prediction the main advantages of system is that huge data storage of past patients record. These medical data helps doctor to examine different pattern in dataset. The pattern used for classification, prediction and diagnosis disease.

\section{RELATED WORK}

A lot of research work has been done on diagnose diabetes using diagnosis system and improve the accuracy of prediction rate. Some of the Research of recent year briefed below.

In the Paper[4] "Fuzzy based modelling for diabetic decision support using Artificial Neural network Author K.Rajeshwari et al, (2011) describe fuzzy model for diabetic diagnostic decision support. The procedure includes combination of fuzzy modelling and artificial neural network architecture. This approach is used for a patient who wares already diabetic .The parameter to be modelled for fuzzy logic ware obtains by interviewing technique. This method was proven to be very efficient and the accuracy was also very good as it work on real time data set.

In this Paper[3] "Detection of diabetic retinopathy using radial basis function "author R.Vijayamadhaeswaran et al.(2011) proposed radial basis function to detect diabetes in retinopathy image. The technique includes combination of two methods such as contextual clustering segmentation method for checking the presence of exudates in the eye and classification of exudates by radial basis function network. The effectiveness of RBF technique is around $96 \%$ The stated RBF technique classifies the segment information of image into hard Exudates provide that the fundus images ware transformed to template condition they are good quality to improve accuracy.

In the Paper[5]"A fuzzy expert System for Heart Disease Diagnosis "Author Ali Adeli et (2010) developed a fuzzy expert system for diagnosis of heart disease. The accuracy value obtain was $94 \%$.

In this paper [7]of "Diagnosis of diabetes Mellitus based on risk Factor "author Sumathy et al.(2010) Proposed method that diagnosed based on risk factor .The suggested system used Artificial neural network (ANN)architecture for classification which had surprised multilayer feed forward network with back propagation learning algorithm. The ANN technique gave better result than other Existing technique.

In this paper[2] of "Improving prediction rate of diabetes Diagnosis using Fuzzy, neural, Case based approach "author MythilTirugnanam,Dr Praveen kumar proposed new approach would called FNC approach using newly design influenced parameter. Technique gave better resultIn this 
paper[1]"Performances evaluation of Levenberg Marquardt Technique in error reduction for Diabetic condition classification" author Nawaz Khan and DharaGaurav(2013) describe the learning algorithms is applied on dynamic constructed neural network to minimize the error by continually training network to until the optimum efficiency level is obtain.Privide optimum output result when applied to dynamically constructed neural network

The above survey indicate different technique used for prediction of diabetes but all system had nuber of problem and prediction rate not appreciable.so new system is proposed that improve prediction rate.

\section{PROPOSED SYSTEM}

The proposed system combination of Artificial neural network and Fuzzy logic. Improve the cluster using Gaussian kernel function.

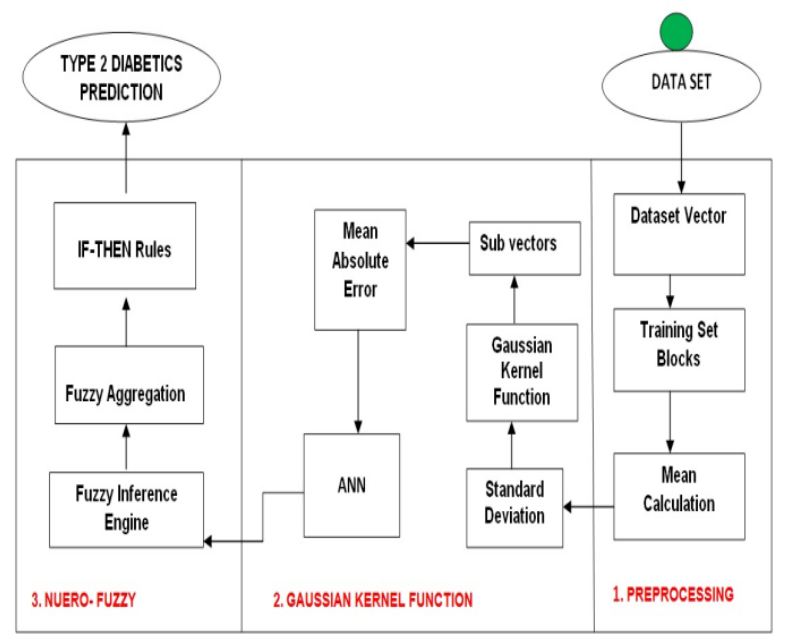

Fig 1.Proposed architecture for diagnosis of diabetes using gauss ion kernel function

\subsection{Preprocessing}

This is the first step of the proposed system. Give the Dataset as input to system. Here medical data taken from UCI repository for Diabetes that is Pima indium Diabetes dataset (PIDD) .In Prepossessing is very important step is performed by removal of noisy. Here we calculate mean for finding missing value and replace missingvalue. Thenpre-processed data subset applied for best result.

\subsection{Applying Gaussian Kernel function}

Classification of data is performed by using gauss ion kernel function.Gaussion kernel distribute data very accurately and fast. First we calculate standard deviation which used by Gaussian function.

\subsection{Applying Artificial neural network}

Artificial neural network is best for pattern recognizatoion.in proposed system the already distributed data is fed to input layer of ANN which consist of $n$ neurans . The hidden Layer operation based on input layer and it consist of $n+1$. The output layer represent either 0 and 1,here 0 used for patient not affected by diabetes and 1 used for patient affected by diabetes. , we examined how many relevant clusters using artificial neural network.

\subsection{Applying fuzzy concept}

Fuzzy is rule based system rule is main function in fuzzy interference system. And quality of result in fuzzy based system on fuzzy rule. Improve better result with the help of fuzzy logic

\subsection{Set Theory}

1. Let $S=\{\}$ be as system for Type 2 Diabetics Prediction

2. Identify Input as $D=\left\{d_{1}, d_{2}, d_{3}\right.$ Where $\mathrm{d}_{\mathrm{i}}=$ dataset Tuples $\mathrm{S}=\{\mathrm{D}\}$

3. Identify $A_{n}$ as Output i.e. Type 2 Diabetics Prediction

$\mathrm{S}=\left\{\mathrm{D}, \mathrm{A}_{\mathrm{n}}\right\}$

4. Identify Process $P$

$\mathrm{S}=\left\{\mathrm{D}, \mathrm{A}_{\mathrm{n}, \mathrm{P}}\right\}$

$\mathrm{P}=\left\{\mathrm{P}_{\mathrm{r}}, \mathrm{G}_{\mathrm{f}}, \mathrm{N}_{\mathrm{n}}, \mathrm{F}_{1}\right\}$

Where

$\mathrm{P}_{\mathrm{r}}=$ Pre-processing

$\mathrm{G}_{\mathrm{f}}=$ Gaussian kernel Function

$\mathrm{N}_{\mathrm{n}}=$ Artificial neuro network

$\mathrm{F}_{1}=$ Fuzzy Logic

5. $S=\left\{D, A_{n}, P_{r}, G_{f}, N_{n}, F_{1}\right\}$

Set description:

\subsubsection{Preprocessing}

Set $\mathrm{P}_{\mathrm{r}}$ :

$\mathrm{P}_{\mathrm{r} 0}=$ get Data set vector

$\mathrm{P}_{\mathrm{r} 1}=$ create Training set Blocks

$\mathrm{P}_{\mathrm{r} 2}=$ Calculate Mean

$\mathrm{P}_{\mathrm{r} 3}=$ Calculate Standard Deviation

\subsubsection{Gaussian Kernel Function \\ Set $\mathrm{G}_{\mathrm{f}}$ :}

$\mathrm{G}_{\mathrm{f} 0}=$ get standard deviation vector

$\mathrm{G}_{\mathrm{f} 1}=$ calculate band width

$\mathrm{G}_{\mathrm{d} 2}=$ Calculate variance

$\mathrm{G}_{\mathrm{f} 3}=$ identify Gaussian factor

\subsubsection{Artificial Nero Network}

Set $\mathrm{N}_{\mathrm{n}}$ :

$\mathrm{N}_{\mathrm{n} 0}=$ Bipolar sigmoid activation function

$\mathrm{N}_{\mathrm{n} 1}=$ Broadcast neutrals

$\mathrm{N}_{\mathrm{n} 2}=$ Calculate Mean absolute error

$\mathrm{N}_{\mathrm{n} 3}=$ Calculate grediances

$\mathrm{N}_{\mathrm{n} 4}=$ get train values

\subsubsection{Fuzzy Logic}

Set $\mathrm{F}_{1}$ :

$\mathrm{F}_{10}=$ Crisp values

$\mathrm{F}_{11}=$ Fuzzyfier

$F_{12}=$ Defuzzyfication

$\mathrm{F}_{13}=$ If-then Rules

$\mathrm{F}_{14}=$ Summary 


\section{DATASET}

The ability to diagnose diabetes early plays an important role for the patient's treatment process. For Experimental purpose dataset downloaded from UCI machine learning dataset repository for Dibetes.The dataset URL is archive.ics.uvi.edu/ml/dataset the World Health Organization proposed the nine attributes. They are shown in below table

\section{Table 1. Diabetes Attribute Set}

\begin{tabular}{|l|l|}
\hline Sr no & Attribute \\
\hline 1 & Number of times pregnant \\
\hline 2 & $\begin{array}{l}\text { Plasma glucose concentration in an oral glucose } \\
\text { tolerance test }\end{array}$ \\
\hline 3 & Diastolic blood pressure $(\mathrm{mm} / \mathrm{Hg})$ \\
\hline 4 & Triceps skin fold thickness $(\mathrm{mm})$ \\
\hline 5 & 2-hour serum insulin $(\mu \mathrm{U} / \mathrm{ml})$ \\
\hline 6 & Body mass index $(\mathrm{kg} / \mathrm{m})$ \\
\hline 7 & Diabetes Pedigree Function \\
\hline 8 & Age $($ year $)$ \\
\hline 9 & Stutus(0-Healthy,1 Diabetes $)$ \\
\hline
\end{tabular}

\section{RESULTS AND DISCUSSIONS}

To show the effectiveness of proposed system some experiments are conducted on java based windows machine using apache tomcat as server. To measure the performance of the system we set the bench mark by selecting the data set of 800 patients of diabetic disease as main measuring protocols of disease detection system.

To determine the performance of the system, we examined how many relevant diseases are identified based on our Neuro-fuzzy k-means classification approach.

To measure this precision and recall are considering as the best measuring techniques. So precision can be defined as the ratio of the number of relevant diseases identified to the total number of irrelevant and relevant diseases identified. It is usually expressed as a percentage. This gives the information about the relative effectiveness of the system.

Whereas Recall is the ratio of the number of relevant diseases are identified to the total number of relevant diseases not identified and it is usually expressed as a percentage. This gives the information about the absolute accuracy of the system.

The advantage of having the two for measures like precision and recall is that one is more important than the other in many circumstances.

For more clarity let we assign

- $\mathrm{A}=$ The number of relevant diseases identified,

- $\mathrm{B}=$ The number of relevant diseases not identified, and

- $\mathrm{C}=$ The number of irrelevant diseases identified.

$$
\text { So, Precision }=(\mathrm{A} /(\mathrm{A}+\mathrm{C}))^{*} 100
$$

And Recall $=(\mathrm{A} /(\mathrm{A}+\mathrm{B})) * 100$

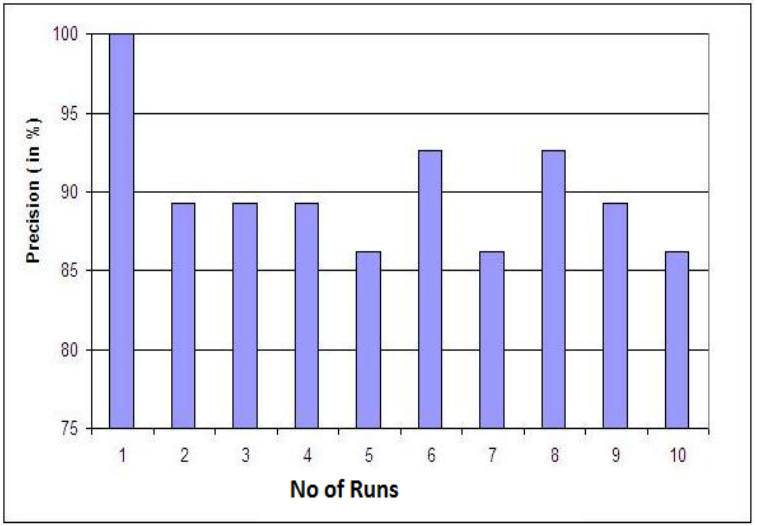

Fig. 2 Average precision of the proposed approach

In Figure 2 we observe that the tendency of average precision for the identified diseases are high compared to other systems

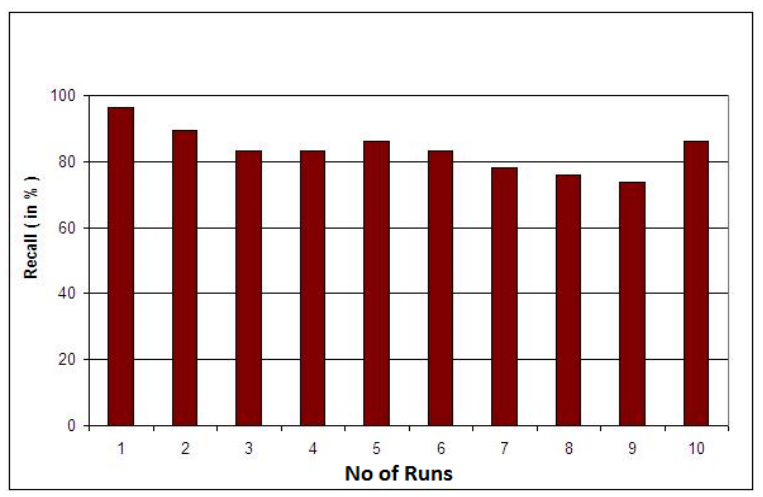

Fig. 3 Average Recall of the proposed approach

In Figure 3, we observe that the tendency of average Recall for the identified diseases are high compared to other system. So this shows that our proposed system is achieving high accuracy than any other method

\section{CONCLUSIONS}

In this paper we have combined Artificial neural network, Fuzzy logic and gauss ion kernel function.

The proposed system takes the entered patients statistics and provides the desired cluster of the data which are actually belongs to diabeticsdisease. We apply gaussion kernel function which used for classification of data accuratly.then it fed to artificial neural network which used for clustering of data.we examined how many relevant clusters are extracted and at what extent they are synced with the given statistics of the patient. This is done using artificial neural network which is blended with the fuzzy logic to extract the cluster. After completing the implementation using this method accuracy of prediction rate is improved.

\section{ACKNOWLEDGMENT}

I would like to thank the researchers as well as publishers for making their resources available and teachers for their guidance. I'm thankful to the authorities of Savitribai Phule University of Pune and concern members of cPGCON2015 conference, organized by , for their constant guidelines and support. I'm also thankful to reviewer for their valuable suggestions and also thank the college authorities for providing the required infrastructure and support. 


\section{REFERENCES}

[1] Nawaz Khan,DharaGaurav,ThomasKandl "Performance Evaluation of Levenberg Marquardt Technique in Error Reduction for Diabetes Condition "International conference on computer Science,ICCS 2013

[2] MythiliThirugnanam,Dr Praveen Kumar "Improving the Prediction Rate of Diabetes using fuzzy ,Neural Network, Case Based (FNC) Approach "Elsevier Transaction1709-1718 International conference on computational Science,ICCS,2012

[3] Me.R.Vijayamadheswaran,Dr.M.Arthanari“ Detection of Diabetic Retinopathy Using Radial Basis Using Radial Basis Function" International journal innovative technology and creative engineering vol 1,pp 40-47, Jan 2011

[4] K.Rajeshwari,VVaithiyanthan "Fuzzy Based Modeling for Diagnostic Decision Support Using Artificial Neural Network" IJCSNS April 2011

[5] Ali adeli,MehdiNeshat "A Fuzzy Expert System for Heart Disease Diagnosis” IMECS ,vol 1,March 2010

[6] Bum JuLee,Boncho $\mathrm{Ku}$ "Prediction of Fasting Plasma Glucose Status Using Anthropometric Measures For Diagnosing TYPE 2 Dibetes"IEEE journal of Biomedical and Health informatics vol 18 March 2014

[7] K kalaiselvi G. m Nasira "A new approach of diagnosis of Diabetes and prediction of cancerusing ANFIS",IEEE computing and communicating technology 2014.pp188190

[8] Velu C.M,K.R Kashwan "Visual data mining Technique and classification of Diabetes patient",IEEE international Advance computing conference(IACC)2013,pp10701075

[9] A.M.Aibinu,M.J.E Salami and A.A.Shafie, "application of modeling Technique to diabetes Diagnosis" IEEE trans Biomed engineering,pp 194-198.2010

[10] AdemKarahoca,dilekKarahoca and Ali Kara "Diagnosis of diabetes using Adaptive Neuro fuzzy interference" IEEE trans 2009

[11] Chang shing Lee and Mei-Hui Wang "a fuzzy Expert system for diabetes decision support application",IEEE trans vol 41,pp 139-153,2011

[12] Asha Gowdak aregowda,M.A.Jayaram , "Integrating Decision tree and ANN for categorizationof diabetes data" International Conference on Computer Aided Engineering December 13-15,2007 Chennai ,India.

[13] Humar K R Novruz "design of hybrid system for the diabetes and heart diseases" expert system with application 2008

[14] UCI machine learning Repository-Centre for machine learning and intelligent system, http://archive.ics.uci.edu

[15] Diabetes mellitus, http://en.wikipedia.org/wiki/Diabetes mellitus 